\title{
TWO WORD RINGS
}

WILLIAM G. LEAVITT

1. Introduction. A ring $R$ is said to be dimensional if for every finitely based right module $M$ over $R$ there is an integer $n$ such that: (i) every basis of $M$ has length $n$, (ii) no independent subset of $M$ has length greater than $n$. If every such module satisfies at least condition (i) then $R$ is said to have the invariant basis property. In a recent paper [1] the author has considered such rings, with particular reference to intrinsic conditions on a general ring to give it one or both of these properties. It is the purpose of this note to construct two examples of rings which will serve to illustrate and sharpen certain results of [1]. The rings to be constructed will be word rings of the type introduced by Malcev [2] (see also [3]).

2. A dimensional ring. In $[1$, Theorem $8(p=2)]$ it is shown that a ring $R$ with unit is dimensional if for each $a, b \in R$ there exist $x, y \in R$, at least one of which is not a left zero divisor, such that $a x+b y=0$. From an examination of the proof of this theorem it is evident that a slightly weaker condition is sufficient. Call a subset $I$ of $R$ integral if it does not include zero and is closed under multiplication, then the following generalization may be stated:

Theorem. $A$ ring $R$ with unit is dimensional if an integral set $I$ exists such that for each $a, b \in R$ there exist $x, y \in R$, at least one of which is a member of $I$, such that $a x+b y=0$.

The ring to be constructed in this section will be shown to obey this theorem while not satisfying any of the usual criteria for the dimensionality (or even invariance of basis length) of a module over the ring. Thus it is noncommutative, has zero divisors (so not imbeddable in a division ring), and does not satisfy either chain condition.

Let $\left\{x_{i}\right\}(i=1,2, \cdots)$ be a countably infinite set of symbols, and let $R$ be the word ring (with unit) formed from the $\left\{x_{i}\right\}$ (that is, $R$ is the set of all polynomials in the $\left\{x_{i}\right\}$ with integers as coeffcients). Consider the set of equations

$$
x_{i}^{m} x_{i}^{n}-x_{j}^{n} x_{i}^{m}=0,
$$

Received by the editors November 13, 1954 and, in revised form, September 30, 1955. 
where $m, n, i$, and $j$ run through all positive integers satisfying: (a) $m+n \geqq 3$, (b) $m \geqq n$, (c) $i \neq j$, (d) if $m=n$ then $i>j$. A two-sided ideal $H$ may be formed generated by the left-hand members of (1) provided it can be shown that a solution exists for the decision problem as to whether or not an $\alpha \in R$ belongs to $H$. Let $S$ be the set $\left\{x_{i}^{m} x_{j}^{n}\right\}$ of all the left-hand words of (1). If each $\alpha \in R$ may in a finite number of steps be transformed using the relations of (1) into a unique normal form $N(\alpha)$ containing no member of $S$, then clearly $\alpha \in H$ if and only if $N(\alpha)=0$. Thus the existence of such an $N(\alpha)$ would provide a solution for the decision problem.

Suppose $\alpha \in R$ and let $z$ be a word of $\alpha$. If every symbol appears in $z$ to the first power then $z$ is already in normal form, so we suppose that at least one symbol appears in $z$ to power 2 or higher. For each such $x_{i}$ of $z$ add the total of all its exponents and consider those $\left\{x_{i}\right\}$ for which this sum is maximum; of these choose the symbol, say $x_{k}$, whose index is largest. Since $z$ contains $x_{k}^{h}$ with $h \geqq 2$ this word may, using (1), be permuted with any other word, and may thus be used to collect together the total power of $x_{k}$ occurring in $z$, and to place it at the right-hand end of the transformed $z$. The remaining portion of the transformed $z$ is then treated in a similar way, using the symbols with successively lower indices, and then successively lower total power. Thus in a finite number of steps all symbols occurring to power 2 or higher have been collected. It is then clear, by the seq uence in which these symbols have been selected, that the resulting word contains no occurrence of a member of $S$. Furthermore, the resulting form is unique for it depends only: (a) for those symbols occurring to power 2 or greater, on the total power of that symbol, (b) for those symbols occurring only to first power, on the initial order of those symbols.

Since each word of $\alpha$ may be reduced in a similar manner, it is clear that for each $\alpha \in R$ we reach in a finite number of steps a unique normal form $N(\alpha)$. Also, it is evident that $N(\alpha+\beta)=N(\alpha)+N(\beta)$ and $N(\alpha \beta)=N[N(\alpha) N(\beta)]$. Since now we have the criterion: $\alpha \in H$ if and only if $N(\alpha)=0, H$ is a two-sided ideal and we may speak of the factor ring $K=R / H$.

This ring is noncommutative $\left(x_{i} x_{j} \neq x_{j} x_{i}\right)$ and since it has zero divisors $\left[x_{i}^{2}\left(x_{i} x_{j}-x_{j} x_{i}\right)=0\right]$ it is not imbeddable in a division ring. Further, it has the proper ascending chain of right ideals $\left(x_{1}\right)$, $\left(x_{1}, x_{2}\right), \cdots$ and the proper descending chain $\left(x_{1}, x_{2}, x_{3}, \cdots\right)$, $\left(x_{2}, x_{3}, \cdots\right), \cdots$. The ring thus does not satisfy any of the usual criteria for a dimensional ring. We shall show, however, that $K$ does satisfy the above theorem, and is therefore a dimensional ring. 
Suppose $\alpha=\alpha\left(x_{i_{1}}, \cdots, x_{i_{h}}\right)$ and $\beta=\beta\left(x_{j_{1}}, \cdots, x_{j_{k}}\right)$. If either $\alpha \gamma=0$ or $\beta \delta=0$ with $\gamma=x_{i_{1}}^{2} \cdots x_{i_{h}}^{2}, \delta=x_{j_{1}}^{2} \cdots x_{j_{k}}^{2}$ then, respectively, we may write $\alpha \gamma+\beta 0=0$ or $\alpha 0+\beta \delta=0$. In all other cases, since multiplication of $\alpha$ by $\gamma$ and of $\beta$ by $\delta$ converts them into commutative polynomials, we may write

$$
\alpha(\gamma \beta \delta)+\beta(-\delta \alpha \gamma)=0 .
$$

Further, since all multiples used are commutative polynomials, and since no product of such polynomials is zero unless one of its factors is zero, the set of all multiples chosen in the above manner is an integral set.

3. A nonimbeddable ring. In another theorem of [1] (Theorem 18) it was shown that a sufficient condition on a ring with unit $R$ that every finitely based module over $R$ have invariant basis number is that the ring be imbeddable in a ring with descending chain condition on right ideals. The ring to be constructed in this section shows that this condition is not necessary.

Let $R$ be the word ring with unit constructed on symbols $a, b$ with rational coefficients. Let $H$ be the set of all multiples of $a b-1$. A member of $R$ is clearly reducible to a unique normal form (not containing $a b$ ) by replacing all occurrences of $a b$ by 1 . Thus $H$ is a two-sided ideal and we have the factor ring $K=R / H$.

The ring $K$ is not imbeddable in a ring $K^{\prime}$ with descending chain conditions on right ideals. For suppose the chain $(b) \supset\left(b^{2}\right) \supset \cdots$ is finite in length, then for some $n$ and some $x \in K^{\prime}$ we have $b^{n+1} x=b^{n}$. But since in $K$ (and thus in $K^{\prime}$ ) $a b=1$, this would imply that $x=a$ and $b a=1$.

It is nevertheless true that all finitely based modules over $K$ have the invariant basis number property. For suppose a module over $K$ exists with bases of lengths $m, n(m>n)$, then $m$ by $n$ and $n$ by $m$ matrices $A, B$ exist such that $A B=I_{m}$. Now all words in elements of $A$ are of form $b^{i} a^{j}$ and we may let $h$ be the largest power of $b$ occurring in $A$. Upon multiplying on the left by $a^{h}$ we then have $A^{\prime} B=a^{h} I_{m}$, where all elements of $A^{\prime}$ are polynomials in $a$. Since the subring of all polynomials in $a$ is an elementary divisor ring, there exist unimodular matrices $P, Q$ such that $P A^{\prime} Q$ is in elementary divisor form. Thus $P A^{\prime} Q Q^{-1} B=a^{h} P$. But the last $m-n$ rows of $P A^{\prime} Q$ are zero so this relation is impossible.

\section{REFERENCES}

1. W. G. Leavitt, Finite dimensional modules, Anais da Academia Brasileira de Ciências, vol. 27 (1955) pp. 241-250. 
2. A. Malcev, On the immersion of an algebraic ring into a field, Math. Ann. vol. 113 (1936-1937) pp. 686-691.

3. J. S. Shepherdson, Inverses and zero divisors in matrix rings, Proc. London Math. Soc. (3) vol. 1 (1951) pp. 71-85.

UNIVERSITY OF NEBRASKA

\section{A SPLITTING THEOREM AND THE PRINCIPAL IDEAL THEOREM FOR SOME INFINITELY GENERATED GROUPS}

\section{EUGENE SCHENKMAN ${ }^{1}$}

The first theorem of this paper is an extension of a splitting theorem for finite groups (cf. [6]) to include periodic groups certain of whose Sylow subgroups contain no elements of infinite height.

The second theorem is an extension of the principal ideal theorem for finitely generated groups (cf. [7]) to include all groups except again that some of the Sylow subgroups contain no elements of infinite height. A counter example will show that this latter restriction is necessary for both theorems.

In the first draft of the paper the second theorem was stated for periodic groups, the proof being based on the first theorem. The present more general statement and proof independent of Theorem 1 are due to the referee to whom I am also indebted for a simplification in the proof of the lemma below.

The Splitting Theorem. As usual $G$ will stand for the group, $G^{\prime}$ for its commutator subgroup, and $G^{*}$ for the intersection of the members $G^{m}$ of the descending series of $G$. $E$ will be the subgroup consisting only of the identity $e$. We let $[a, b]$ stand for $a b a^{-1} b^{-1}$ and refer the reader to [6] for some of the standard identities on commutators that will be needed. In particular we shall need that $[x, h g]=[x, h][x, g]$ if $h$ and $g$ are in an Abelian normal subgroup; and $[x, h z]=[x, h]$ if $[x, z]=e$.

Lemma. Let $G$ be a p-group with no elements of infinite height; let $G / G^{*}$ be finite and let $G^{*}$ be Abelian. Then $G^{*}$ consists only of the identity and $G$ is finite.

Presented to the Society, September 2, 1955; received by the editors May 31, 1955.

${ }^{1}$ I wish to thank Professors Beaumont, Curtis, Reiner, and Swift for interesting discussions during the preparation of this paper. Work was supported by the Office of Ordnance Research United States Army Contract No. DA-36-034-ORD-1622RD. 